\title{
Arithmetic Study on Computer Generated Hologram Based on Diffraction Theory
}

\author{
Yu Jie \\ Xiamen University TKK College, lecturer, Zhangzhou, Fujian, 363105
}

Keywords: diffraction theory; computer; generated hologram; phase modulation; algorithm

\begin{abstract}
. based on the diffraction theory, this paper analyses the brand new algorithm of computer generated hologram, and puts forward a brand new computing method, phase-only hologram computing method based on Fourier transform of fractional order, with the support of such theories as Huygens-Fresnel principle, and Helmholtz Kirchhoff integral theorem, which greatly avoids the influence of zero-level diffraction on reconstructed image; this paper also proves that Fourier hologram of fractional order can reconstruct images without the influence of zero-level diffraction on any plane behind lens with experiment, which has certain practical application.
\end{abstract}

\section{Introduction}

Computer generated hologram has certain features as easy to make, convenient to store and transfer, able to compute and display virtual objects, which has gradually replaced generated hologram through optical interference techniques, which becomes an important basis of realizing dynamic holographic 3D display. This paper makes research on the computing method of computer generated hologram, aiming to solve the problems of generated hologram in computers and display through the brand new computing methods based on diffraction theory, so as to elevate the practicability of computer generated hologram in holographic display.

\section{Models and basic theories of computer generated hologram}

The model of generated hologram is shown in Figure 1; holographic plane records the light intensity of the emission or reflection light waves in the scenes after optical interference and the light intensity contains the phase information of object light waves. As computer can only deal with discrete data, in the making process of computer generated holograph, the space object can be taken as the set of a series of self-luminous dimensional target points.

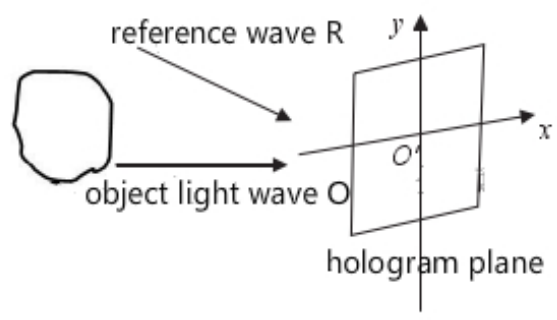

Figure 1 Sketch map of hologram formation

Suppose the space object is composed of $\mathrm{N}$ discrete luminous points and each point on the hologram plane can be lighted up by the discrete waves emitted by each object point; the complex amplitude of any point on the hologram place can be expressed as:

$$
u(x, y)=\sum_{n=1}^{N} a_{n} \exp \left(j \frac{2 \pi}{\lambda} r_{n}+j \varphi_{n}\right)
$$

In the formula above, $\lambda$ is for wave length, $\varphi_{n}$ for initial phase, $a_{n}$ for the diffraction light wave amplitude of the object point $\left(x_{n}, y_{n}, z_{n}\right)$, and $r_{n}$ for the distance from object point to 
hologram plane $(x, y)$.

$$
r_{n}=\sqrt{\left(x_{n}-x\right)^{2}+\left(y_{n}-y\right)^{2}+z_{n}^{2}}
$$

After the superposition of the reference light and the hologram of each object point, we can have hologram of multiple object points; the on the light intensity distribution of interference light intensity can be expressed as:

$$
I(x, y)=A+\sum_{n=1}^{N} a_{n} \cos \left(\frac{2 \pi}{\lambda} r_{n}+j \varphi_{n}^{\prime}\right)
$$

After the completion of the optical field distribution on the computing holographic plane, the holographic coding can be calculated, and the hologram transmittance function can be coded; a complete computing mechanism hologram can be formed after quantification and unification.

\section{Related theory and formula of diffraction theory}

The main job of hologram computing is to calculate the diffraction propagation process of light wave from objects to holographic plane; the diffraction calculation process is mainly calculated with high approximation based on the theory of diffraction of light in the space. Therefore, it is necessary for you to gain some knowledge on the diffraction theory and the diffraction calculations.

\section{Huggens-Fresnel principle}

Huggens-Fresnel principle is the basic principle to explain the propagation law of light based on wave theory. It can be interpreted as: any point on wave front in the traveling can be regarded as the new secondary wave source, and the envelope plane formed by many secondary waves emitted from different points on the wave train plane is the new wave plane that the original wave propagates to. Fresnel diffraction diagram and the calculation formula of planar aperture is shown below

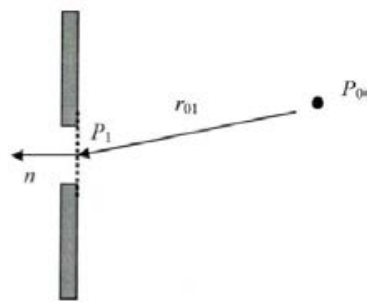

Figure 2 Diffraction sketch map of planar aperture

$$
U\left(P_{0}\right)=\frac{1}{i \lambda} \int_{\Sigma} \int U\left(P_{1}\right) \frac{\exp \left(i K r_{01}\right)}{r_{01}} \cos \theta d s
$$

Simple diffraction patterns can be calculated with formula (4), which is the geometrical relationship on the rectangular coordinate system; diffraction aperture $\Sigma$ is in the plane of $(\xi, \eta)$; light waves propagate through aperture; the optical field distribution of complex amplitude of the plane $(x, y)$ which is parallel to plane $(\xi, \eta)$ with the distance of $z$ can be calculated; $z$ axis goes through the original points of two planes, which can be seen in Figure 3: 


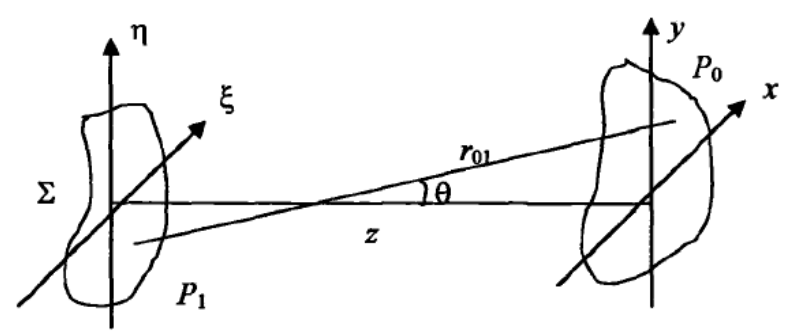

Figure 3 Sketch map of geometrical relationship of diffraction

Through steps of calculation, the ultimate expression of Fresnel diffraction can be gained, which is generally called as Fresnel integration:

$$
U(x, y)=\frac{e^{i K z}}{i \lambda z} \exp \left[\frac{i k}{2 z}\left(x^{2}+y^{2}\right)\right] \iint U(\xi, \eta) \exp \left[\frac{i k}{2 z}\left(\xi^{2}+\eta^{2}\right)\right] \exp \left[\frac{-i 2 \pi}{\lambda z}(x \xi+y \eta)\right] d \xi d \eta
$$

\section{Helmholtz Kirchhoff integral theorem}

This is what Kirchhoff deduced in strictly monochromatic scalar wave under the condition of uniform medium according to the principle of Huygens - Fresnel principle: "the light disturbance of some point is the superposition of wavelets emitted from certain plane between the point and the light source"; therefore, this is called as the Helmholtz Kirchhoff integral theorem.

$$
U(P)=\frac{1}{4 \pi} \int_{S} \int\left[U \frac{\partial}{\partial_{n}}\left[\frac{e^{i k l}}{l}\right]-\frac{e^{i k l}}{l} \frac{\partial U}{\partial_{n}}\right] d S
$$

Similarly, the formula above is also the solution of Helmholtz equation $\left(\Delta^{2}+k^{2}\right) U=0$, which is suitable for the homogeneous medium of monochromatic scalar wave. The common form based on Helmholtz integral theorem is:

$$
U(P, t)=\frac{1}{4 \pi} \iint_{s}\left\{[V] \frac{\partial}{\partial_{n}}\left[\frac{1}{l}\right]-\frac{l}{c l} \frac{\partial_{l}}{\partial_{n}}\left[\frac{\partial V}{\partial_{n}}\right]-\frac{1}{l}\left[\frac{\partial V}{\partial_{n}}\right]\right\} d S
$$

However, the diffraction integral formula is:

$$
U(P)=\frac{1}{i \lambda} \int_{W} \int_{0}(Q) \frac{e^{i k r}}{r}\left[\frac{\cos \theta_{0}+\cos \theta}{2}\right] d S
$$

\section{Pure phase hologram based on fractional Fourier transform is calculated}

Fractional Fourier transform was first in 1980 by Namias, mainly to solve the partial differential equation of quantum mechanics, with the mathematical formula of:

$$
\begin{aligned}
& F(u)=C_{p} \int f(x) \exp \left[i \pi\left(x^{2} \cot \phi-2 x u \cdot \csc \phi\right)\right] d x \\
& =C_{p} \int f(x) \exp \left[i \pi\left(x^{2} \cot (a \pi / 2)-2 x u \cdot \csc (a \pi / 2)+u^{2} \cot (a \pi / 2)\right)\right] d x
\end{aligned}
$$

In 1987, Mcbride and kerr et al. introduced Fractional Fourier transform into optics, combining fractional Fourier transform with optics, pushing the development of fractional Fourier optics forward. 


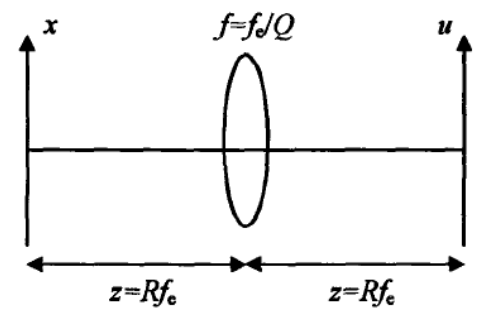

Figure 4 Fractional Fourier transform optical system (single lens)

The formula of light from $\mathrm{x}_{0}$ plane to $\mathrm{x}$ plane is:

$$
F(u)=\int f(x) \cdot \exp \left[i \pi\left(\frac{x^{2}}{\lambda f_{e} \tan (a \pi / 2)}+\frac{u^{2}}{\lambda f_{e} \tan (a \pi / 2)}=\frac{2 u x}{\lambda f_{e} \sin (a \pi / 2)}\right)\right] d x
$$

The hologram gained from the calculation of improved algorithm of GS Fourier transform (modified GS) based on the score level of fractional Fourier transform put forward above, is called fractional Fourier hologram.

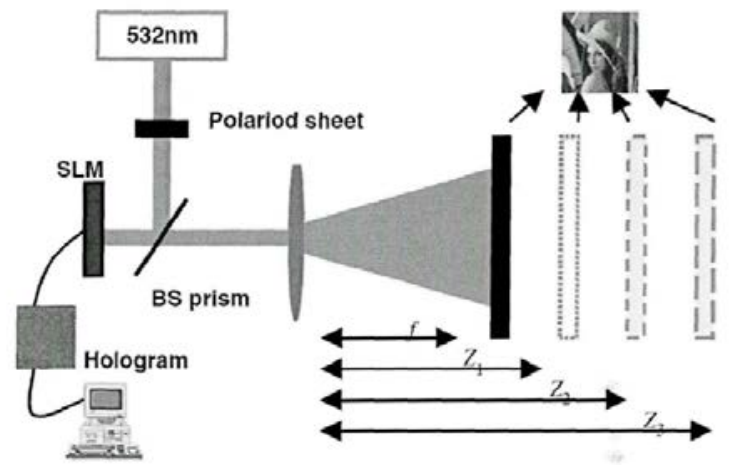

Figure 5: Holographic optical sketch map based on fractional Fourier transform

Based on the analysis above, in the described optical system of the fractional order Fourier transform, when the lens focal length is fixed, order a and imaging distance $\mathrm{z}$ is one-to-one, different order number corresponding to different distances; due to the characteristics of the fractional Fourier transform, the images can be projected to the arbitrary plane after the lens. Using three different order numbers a $=1.1$, a $2=1.25$, and a $=1.4$, the projection results can be gained below:

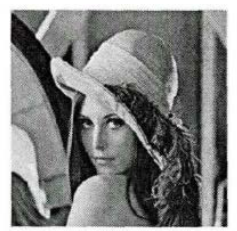

原图像

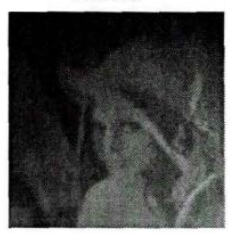

$a=1.25$

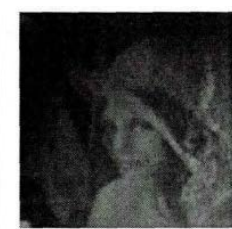

$a=1.1$

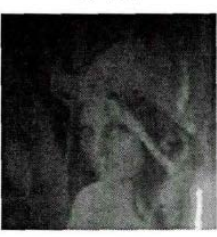

$a=1.4$

Figure 6: Holographic reconstructed image of different stages

The image at the top left corner is the original image, with the gray scale of 256; three other images are the projection images of fractional Fourier hologram of three different orders; it can be 
seen that, the fractional Fourier hologram can successfully project the image on the corresponding different distances; the reconstructed image quality is of no difference, which can clearly reconstruct the original images. In this case, compared with the traditional Fourier projection, this new fractional Fourier holographic projection method's advantage is that it can realize the image projection reconstruction on the arbitrary plane behind the lens, unlike Fourier hologram which can only project image behind a back focal plane.

\section{Conclusion}

To sum up, the fractional Fourier transform can be used to calculate the diffraction propagation from object to the surface of the holographic plane, and pure phase fractional Fourier hologram can be gained through the combination with the iteration algorithm; the reconstruction images can be displayed on the arbitrary plane. Using this feature, the reconstruction image can avoid the influence of the zero-order diffraction through placing a filter in the lens focal plane to block off the zero-order diffraction noise. Optical experimental results proved that the fractional Fourier hologram can reconstruct the images without the influence of zero-order diffraction on arbitrary plane behind the lens. Moreover, compared with using an iterative algorithm, the error of the reconstructed images of pure phase hologram generated by this algorithm is smaller; the experimental results show that as to the 256 gray-scale image, the reconstruction image of root mean square error (RMS) values could drop by about 45 gray-scale, which can be applied to holographic retina display; if we take the eye lens as the corresponding lens in fractional Fourier transform system, the effect of augmented reality can be achieved through directly watching the fractional Fourier hologram.

\section{References}

[1] Chang Chenliang. Computer Hologram Algorithm Research Based on Diffraction Theory [D]. Doctoral Dissertation of Southeast University, 2015

[2] Chen Huirong, Fu Shenghao et al. The Fast Algorithm of A Kind of Computer Generated Hologram[J]. Computer Engineering and Applications, 2013, 49(18): 142-144

[3] Zhu Xiangzhu, Zeng Yangsu. The Interpretation and Application of Diffraction Theory Formula[J]. College Physics, 2007,26(10): 27-30

[4] Jia Fang. Study on the Digital Holographic Principle and Application [D]. Northwest University Master's Thesis, 2008

[5] Ding Dawei. Study on the Computer Hologram and Its Digital Reconstruction [D]. Anhui University Master's Thesis, 2004

[6] Li Guanghua. Research on the Circular Aperture Near-field Diffraction Characteristics and Different Diffraction Theory Methods [D]. Shandong Normal University Master's Thesis, 2013

[7] Chen Huirong, Fu Shenghao, Wang Yuanqing. Hologram Rapid Generation of Active Holographic Display [J]. Optoelectronic Component, 2014, 41(1): 48-53

[8] Sheng Zhaoxuan, Sun Xinli. Fractional Fourier Transform Computer-generated Holography [J]. Optical Technology, 2008, 34(1): 156-158. 